\title{
RETROSPECTIVE ANALYSIS OF CHRONIC MYELOID LEUKEMIA PATIENTS IN TRAKYA UNIVERSITY SCHOOL OF MEDICINE
}

\author{
Fatih Erkan Akay ${ }^{1}$, Beliz Koçyiğit ${ }^{1}$, Berfin Tan ${ }^{1}$, Emine İkbal Atlı ${ }^{2}$, Volkan Bas ${ }^{3}$, Hakkı Onur Kırkızlar ${ }^{3}$ \\ ${ }^{1}$ Trakya University School of Medicine, Edirne, TURKEY \\ ${ }^{2}$ Department of Medical Genetics, Trakya Univeristy School of Medicine, Edirne, TURKEY \\ ${ }^{3}$ Division of Hematology, Department of Internal Medicine, Trakya University School of Medicine, Edirne, TURKEY
}

\begin{abstract}
Aims: This study aims to establish the data; including demographic features, molecular response status, disease characteristics, and survival rate of chronic myeloid leukemia patients treated with tyrosine kinase inhibitors in Trakya University School of Medicine. Methods: In this study, the data of 102 patients over 18 years old who were diagnosed with chronic myeloid leukemia in Trakya University School of Medicine between January 2003-October 2019 were analyzed retrospectively. Data was analyzed using SPSS 23.0.0.0. Results: The total number of patients in the study was 102. There were 95 (93.1\%) patients on chronic phase and $6(5.9 \%)$ patients on accelerated phase. Eighty-three (81.4\%) of the patients had at least a major molecular response and 17 (16.7\%) patients could not achieve at least a major molecular response. Conclusion: As our study showed, first line treatment with imatinib may not be enough for some of the patients to recover and therefore different TKIs such as dasatinib, nilotinib, bosutinib, and ponatinib are being used in the treatment of CML. Inadequate response, drug side effects and incompliances are the causes of switching the drug choice. Further studies are needed to thoroughly reveal the epidemiology and treatment regimens.
\end{abstract} Keywords: Chronic myeloid leukemia, tyrosine kinase, neoplasms

\section{INTRODUCTION}

Chronic myeloid leukemia (CML) is a myeloproliferative neoplasm where most of the time a single, pluripotent hematopoietic stem cell develops a Philadelphia chromosome $(\mathrm{t} 9 ; 22)$ resulting with a clonal overgrowth of myeloid precursor cells (1). The pathogenesis that plays a crucial role in CML is irrepressible tyrosine kinase activity which is a by-product of translocation between 9 and 22 chromosomes giving rise to fusion genes BCR-ABL1 (1). The clinical presentation may be seen in 3 different phases: chronic, accelerated and blastic. Most patients are diagnosed at the chronic phase of the disease and if left untreated may progress to accelerated or blastic phases, which have poorer prognosis (2). Chronic myeloid leukemia represents about $15 \%$ of adults leukemias, the incidence is approximately $1-2 / 100,000$ per year. CML may occur in all age groups, however, the median age of disease is 67 (3).
Diagnosis is made by history, physical examination and laboratory tests which comprise, which comprises spleen palpation, complete blood count, hepatitis panel and chemistry profiles. Bone marrow biopsy and aspirate are used for morphological, molecular and cytogenetic evaluation. However, diagnosis must be approved cytogenetically by using fluorescence in situ hybridization (FISH) which direclty detects the translocation ( $\mathrm{t} 9: 22)$ and quantitative reverse transcriptase-polymerase chain reaction (RT-PCR) that shows the levels of BCR-ABL1 in peripheral blood or bone marrow cells (4).

The European LeukemiaNet and National Comprehensive Cancer Network are the most common guidelines used for the diagnosis and management of CML patients in daily practice $(5,6)$. After patients have been diagnosed and started their first-line Tyrosine Kinase Inhibitor (TKI) treatment they are followed up on their 3rd months where a hematological response (Leukocyte $<10 \times 10^{9} / \mathrm{L}$, no immature myeloid precursors, basophils 
$<5 \%$, platelet count $<450 \times 10^{9} / \mathrm{L}$, spleen non-palpable) is expected. In addition, quantification of BCR-ABL1 from peripheral blood is necessary every 3 months. Having an early molecular response (MR) at 3 months (BCR-ABL1 $<10 \%)$ is a predictive biomarker of later on achieving a deep molecular response (7). Intervals are prolonged until the 12th month of disease expecting a major molecular response (MMR) (BCR-ABL1 0.1\%, 3 log reduction from standardized baseline). Patients' relative risk scores are calculated using simple hematological and clinical data collected before any treatment. For assessing prognostic and predictive implications; Sokal, Euro and European Treatment of Outcome Study (EUTOS) scores are used (8).

First-line therapy options are first-generation (imatinib) and second-generation (nilotinib and dasatinib) TKIs. Second-line therapy options consists of second-generation TKIs (nilotinib, dasatinib, and bosutinib) and third-generation TKI, ponatinib, which is also used in third-line therapy as well as for patients with T315I mutation associated with pan-TKI resistance (8). Later on, with the introduction of imatinib, being the first targeted therapy used in treating CML patients, number of patients applying to allogenic stem cell transplantation underwent a significant decrease, however, it is still an important therapeutic option for the patients who failed at least 2 TKIs or have T315I mutation (8).

Before the 2000s, treatment strategy for CML was limited to interferon-alpha (IFN-alpha), chemotherapy, hydroxyurea and allogeneic stem cell transplantation which was the most efficient treatment strategy (9). Later on, treatment protocol completely changed with the introduction of imatinib, being the first targeted therapy used in treating CML patients (10). One of the largest phase III randomized trials done known as International Randomized Study of Interferon and STI571 (IRIS) had a major impact on providing the necessary information about the scientific and clinical background for treating $\mathrm{Ph}(+)$ leukemias with imatinib (10). Complete cytogenetic response rate for the patients treated with imatinib was $94 \%$ in contrast to patients that were administered IFN-alpha at the 19th month of median follow-up (10). Overall, the superiority of treating CML patients with imatinib has been proved in other prospective and retrospective studies as well (11-13).

This study aims to establish the data; including demographic features, molecular response status, disease characteristics, and survival rate of CML patients treated with TKI in Trakya University School of Medicine which is a tertiary center of the region and make a contribution to the database of CML patients in Turkey.

\section{MATERIAL AND METHODS}

This retrospective study was approved by the Scientific Research Ethics Committee of Trakya University School of Medicine (Protocol Code: TUTF-BAEK2019/359). Informed consent was obtained from all subjects. In this study, the data of all patients over 18 years old who were diagnosed with CML in Trakya University School of Medicine between January 2003-October 2019 were analyzed retrospectively.

There were 102 patients where their demographic data, phase of the disease, total follow-up, durations of their TKI treatments, major molecular response, reason to switch treatments and last treatment they were on were congregated from the archives of Trakya University School of Medicine.

Data was analyzed using SPSS 23.0.0.0. Continuous variables (age, total follow-up and durations of their TKI treatments) were tested for normal distribution with Shapiro-Wilk Test. Normal distribution was observed only for the duration of 4th line TKI treatment which was presented as mean and standard deviation. Non-normal distribution was observed for the rest. Thus, descriptive statistics for those are presented as median and inter-quantile range (IQR). Categorical data (gender, phase of the disease, major molecular response, distribution of TKI treatments, reasons for switching TKIs) are presented as numbers and percentages.

\section{RESULTS}

In this retrospective study 102 patients with the diagnosis of CML were included. 60 (58.8\%) patients were male and $42(41.2 \%)$ were female. There were 95 (93.1\%) patients on chronic phase and 6 (5.9\%) patients on accelerated phase, with 1 missing data. Eighty-three $(81.4 \%)$ of the patients had at least major molecular response and $17(16.7 \%)$ patients could not achieve at least a major molecular response, there were 2 missing data. All patients (102) in our study were admitted imatinib as the first-line treatment.

Number of patients, summary statistics of parametric (duration of 4 th TKI treatment) and non-parametric variables (age, age of diagnosis, total median follow-up and duration of 1st, 2nd and 3rd TKI treatments) are presented in Table 1. Summary of categorical data (gender, phase of the disease, major molecular response, distribution TKI treatments, reasons for switching TKIs) are presented in Table 2.

In median total follow-up time of 86 (88.2\%) month, 20 patients were found to be deceased, with 7 deaths being CML-related and remaining 5 deaths being non-CML-related. 
Table 1: Summary statistics of parametric and non-parametric variables.

\section{Number of patients*}

Current age of patients (years)

Age of the patients at the time of diagnosis (years)

Total follow-up (months)

Duration of $1^{\text {st }}$ line TKI treatment

(months)

Duration of $2^{\text {nd }}$ line TKI treatment

(months)

Duration of $3^{\text {rd }}$ line TKI treatment (months)

Duration of $4^{\text {th }}$ line TKI treatment

(months)**

Median $(I Q R)$

$\begin{array}{lr} & 62.00(26) \\ & 51.98(23) \\ & \\ & 86.00(84) \\ 44 & 15.00(39) \\ 21 & 16.00(19) \\ 9 & 8.00(8) \\ 4 & 20.0 \pm 19.408\end{array}$

${ }^{*}$ All 102 patients started off with Imatinib as the first line treatment, these are the number of patients who were switched to next line treatment and their durations.

TKI: Tyrosine Kinase Inhibitor, IQR: Inter-quartile Range

${ }^{*}$ Since the data of duration of the 4th line TKI treatment is normally distributed, it is given as mean \pm standard deviation.

Table 2: Summary statistics of categorical variables.

\begin{tabular}{|c|c|c|}
\hline \multirow[t]{2}{*}{ Gender } & Male & $60(58.8)$ \\
\hline & Female & $42(41.2)$ \\
\hline \multirow[t]{3}{*}{ Phase } & Chronic & $95(93.1)$ \\
\hline & Accelerated & $6(5.9)$ \\
\hline & $N / A$ & 1 \\
\hline \multirow[t]{3}{*}{ Distribution of $2^{\text {nd }}$ line TKI treatments } & Dasatinib & $17(38.6)$ \\
\hline & Nilotinib & $26(59.1)$ \\
\hline & $\boldsymbol{N} / \boldsymbol{A}$ & 1 \\
\hline \multirow[t]{3}{*}{ Distribution of $3^{\text {rd }}$ line TKI treatments } & Dasatinib & $8(38.1)$ \\
\hline & Nilotinib & $10(47.6)$ \\
\hline & Bosutinib & $3(14.3)$ \\
\hline \multirow[t]{3}{*}{ Reasons to switch $2^{\text {nd }}$ line TKI treatment } & Side effect & $5(11.4)$ \\
\hline & Loss of MR & $37(84.1)$ \\
\hline & Incoordination & $2(4.5)$ \\
\hline \multirow[t]{4}{*}{ Reasons to switch $3^{\text {rd }}$ line TKI treatment } & Side effect & $9(39.1)$ \\
\hline & Loss of MR & $12(52.2)$ \\
\hline & Incoordination & $1(4.3)$ \\
\hline & Refractory & $1(4.3)$ \\
\hline \multirow[t]{9}{*}{ Last treatment } & Dasatinib & $9(8.8)$ \\
\hline & Nilotinib & $22(21.6)$ \\
\hline & Bosutinib & $4(3.9)$ \\
\hline & Imatinib & $57(55.9)$ \\
\hline & Ponatinib & $4(3.9)$ \\
\hline & Hydroxyurea & $2(2.0)$ \\
\hline & Cytarabine & $1(1.0)$ \\
\hline & No drug & $1(1.0)$ \\
\hline & $N / A$ & 2 \\
\hline \multirow{2}{*}{$\begin{array}{l}\text { Major molecular response }(B C R-A B L 1 \\
\leq 0.1 \%\end{array}$} & Responsive & $83(81.4)$ \\
\hline & $\begin{array}{l}\text { No response } \\
\text { N/A }\end{array}$ & $\begin{array}{l}17(16.7) \\
2(1.96)\end{array}$ \\
\hline \multirow[t]{4}{*}{ Survival status } & Alive & $90(88.24)$ \\
\hline & Deceased & $12(11.76)$ \\
\hline & $C M L$-related & $7(6.8)$ \\
\hline & Non-CML-related & $5(4.9)$ \\
\hline
\end{tabular}




\section{DISCUSSION}

Before 2013, epidemiological data from the End Results Program of United States National Cancer Institute were used in daily practice, however with the Turkish CML study, which was a multicentral study with 1133 patients, Turkey managed to establish its database and found the median age for Turkish CML patients to be $46.1 \pm 14.8(9,14)$. Our study has found the mean age of CML patients to be 51.98 years. This proves the need for similar studies to be conducted since different genetic and environmental factors can make up different mean ages. These demographic data are also important for different clinical approaches in daily practice. Since there are higher rates of durable complete molecular response with second-generation TKIs, second-generation TKIs may be considered in younger patients (e.g. $<50$ years) in comparison to older patients. This could lead to potential molecular responses where the patients may end up with the discontinuation of TKI treatments (15).

According to the previous studies, most patients diagnosed with CML present themselves in the chronic phase, however, 5-7\% may advance to accelerated or blastic phases $(9,16)$. Our data was in line with the Turkish CML study, during the initial diagnostic evaluations, where $93.1 \%$ of patients were in the chronic phase whereas $5.9 \%$ of patients were in the accelerated phase (9).

All the patients $(\mathrm{n}=102)$ in our study used imatinib as the first-line of TKI treatments with no regards to the phase of the disease. In IRIS study, only $55 \%$ of the patients were remained on imatinib in 8 year follow-up time (15). This highlighted the need for supplementary treatment options for patients who had to switch their imatinib treatments, which led to the development of second-generation TKIs.

In our study, due to several reasons such as side effects, loss of molecular response and patient incompliance; imatinib was switched to dasatinib or nilotinib in 44 patients. In the study carried out by Şahin et al. (9), $90.8 \%$ of patients had to have their imatinib treatment altered due to their loss of molecular response and 9.2\% had it altered due to drug intolerance. In comparison, $44.11 \%$ of the patients in our study had to switch their imatinib treatment due to inadequate response, side effects or their incompliances.

The study conducted by Russo et al. (17) with 82 CML patients, 34 had nilotinib and 48 of them had dasatinib as their second line TKIs, with prior TKI being imatinib in all patients. In the previously-mentioned Turkish CML study, the first choice after imatinib tre- atment in 194 patients was dasatinib, in 138 patients the first choice was nilotinib and 114 patients had to use both nilotinib and dasatinib during their treatment (9). In our study, out of 44 patients who had to switch first-line TKI treatment, 17 of them had dasatinib and 26 of them had nilotinib as a part of their second-line TKI treatment. Nine of the patients receiving second-line TKI treatment had their treatment switched due to generated side effects, 12 of the patients as a result of a loss in molecular response, 2 of the patients because of incompliances and 1 of the patient had to switch their treatment due to refraction.

Comparing our data concerning the distribution of third-line TKI treatments, while 3 out of 21 patients in our study used bosutinib as their third-line TKI treatment; in the study of Khoury et al. (18) 115 out of 118 patients used bosutinib in third-line TKI treatment. Moreover, 10 patients in our study used nilotinib and 8 patients used dasatinib.

Furthermore, 83 patients enrolled in our study had an MMR following their last treatments where 17 patients had less than MMR. In the IRIS study, patients who had been assigned to take imatinib had a 10-year MMR rate of 93\% (15). In the study conducted by Şahin et al. (9) evaluation of molecular response was not carried out due to a lack of available data in the majority of patients.

In a median total follow-up time of 86 (88.2\%) months, total number of 12 patients was found to be deceased, where 7 deaths were associated with CML and 5 deaths were non-CML-related. Causes of CML-related deaths are included but not limited to cardiac complications, blastic transformation, incompliance with treatment plans. In the IRIS study, for the patients who had been assigned to use imatinib, the estimated survival rate was $83.3 \%$ (15).

The main limitations in our study were the lack of data in molecular (RT-PCR and BCR-ABL1), cytogenetic (FISH) and hematologic responses in the following 3rd, 6th and 12th months of patients' treatment, which are highly important in making decisions when to switch their treatments with the addition to planning future treatment strategies. Our study also lacks the patients' clinical risk scores such as Sokal, Hasford and EUTOS due to the missing data in patients' files.

In conclusion, CML is a myeloproliferative neoplasm which is seen mostly in older adults. As our study showed, first line treatment with imatinib may not be enough for some of the patients to recover and therefore different TKIs such as dasatinib, nilotinib, bosutinib, and ponatinib are being used in the treatment of CML. Inadequate response, drug side effects and incomplian- 
ces are the causes of switching the drug choice. Further studies are needed to thoroughly reveal the epidemiology and treatment regimens.

Ethics Committee Approval: This retrospective study was approved by the Scientific Research Ethics Committee of Trakya University School of Medicine (Protocol Code: TUTF-BAEK2019/359).

Informed Consent: Informed consent was obtained from all subjects. Conflict of Interest: The authors declared no conflict of interest.

Author contributions: Concept: FEA, BK, BT, HOK Supervision: FEA, BK, BT, HOK Resources: FE, BK, BT, EİA, VB, HOK Materials: FEA, BK, BT, EİA, VB, HOK Data collection and/or processing: FEA, BK, BT, EİA, VB, HOK Analysis and/or Interpretation: FEA, BK, BT, HOK Literature Search: FEA, BK, HOK Writing Manuscript: FEA, BK, BT, HOK Critical Review: FEA, BK, BT, HOK

Financial disclosure: The authors declared that this study received no financial support.

Editor-in-chief's Note: Three of the authors of this article; Fatih Erkan Akay, Beliz Koçyiğit and Berfin Tan are members of the editorial board of Turkish Medical Student Journal. However, they did not take place in any stage on the editorial decision of the manuscript. The editors who evaluated this manuscript are from another institutions.

\section{REFERENCES}

1. Faderl S, Talpaz M, Estrov Z et al. The biology of chronic myeloid leukemia. New England Journal of Medicine 1999;341(3):164-72.

2. Kurzrock G, Gutterman J, Talpaz M. The molecular genetics of Philadelphia chromosome-positive leukemias. N Engl J Med 1988;319:990-8.

3. Siegel RL, Miller KD, Jemal A. Cancer statistics, 2018. CA Cancer J Clin 2018;68:7-30.

4. Braziel RM, Shipp MA, Feldman AL et al. Molecular diagnostics. Hematology 2003;(1):279-93.

5. Baccarani M, Deininger MW, Rosti G et al. European LeukemiaNet recommendations for the management of chronic myeloid leukemia: 2013. Blood 2013;122(6):872-84.

6. Radich JP, Deininger M, Abboud CN et al. Chronic myeloid leukemia, version 1.2019, NCCN clinical practice guidelines in oncology. Journal of the National Comprehensive Cancer Network 2019;16:1108-35.

7. Hanfstein B, Müller MC, Hehlmann R et al. Early molecular and cytogenetic response is predictive for long-term rogression-free and overall survival in chronic myeloid leukemia (CML). Leukemia 2012;26:2096-102.

8. Hochhaus A, Saussele S, Rosti G et al. Chronic myeloid leukaemia: ESMO clinical practice guidelines for diagnosis, treatment and follow-up. Annals of Oncology 2017;28(4):41-51.

9. Sahin F, Saydam G, Comert M et al. Turkish chronic myeloid leukemia study: retrospective sectional analysis of CML patients. Turk J Hematol 2013;30:351-8.
10. O’Brien SG, Guilhot F, Larson RA et al. Imatinib compared with interferon and low-dose cytarabine for newly diagnosed chronic-phase chronic myeloid leukemia. N Engl J Med 2003;348:9941004.

11. Druker BJ, Guilhot F, O'Brien SG et al. Five-year follow-up of patients receiving imatinib for chronic myeloid leukemia. $\mathrm{N}$ Engl J Med 2006;355:2408-17.

12. Roy L, Guilhot J, Krahnke T et al. Survival advantage from imatinib compared with combination interferon-alpha plus cytarabine in chronic-phase chronic myelogenous leukemia: historical comparison between two phase 3 trials. Blood 2006;108:1478-84.

13. Bittencourt H, Funke V, Fogliatto L et al. Imatinib mesylate versus allogeneic BMT for patients with chronic myeloid leukemia in first chronic phase. Bone Marrow Transplant 2008;42:597-600.

14. National Cancer Institute. Surveillance, Epidemiology, and End Results Program. (online) (cited 2019 August 5). Available from: URL:https://seer.cancer.gov.

15. Jabbour E, Kantarjian H. Chronic myeloid leukemia: 2018 update on diagnosis, therapy and monitoring. Am J Hematol 2018;93(3):44259.

16. Shanmuganathan N, Hiwase DK, Ross DM. Treatment of chronic myeloid leukemia: assessing risk, monitoring response, and optimizing outcome. Leukemia \& Lymphoma 2017;58(12):2799-810.

17. Russo RA, Breccia M, Abruzzese E et al. Outcome of 82 chronic myeloid leukemia patients treated with nilotinib or dasatinib after failure of two prior tyrosine kinase inhibitors. Haematologica 2013;98:399-403.

18. Khoury HJ, Cortes JE, Kantarjian HM et al. Bosutinib is active in chronic phase chronic myeloid leukemia after imatinib and dasatinib and/or nilotinib therapy failure. Blood 2012;119:3403-12. 